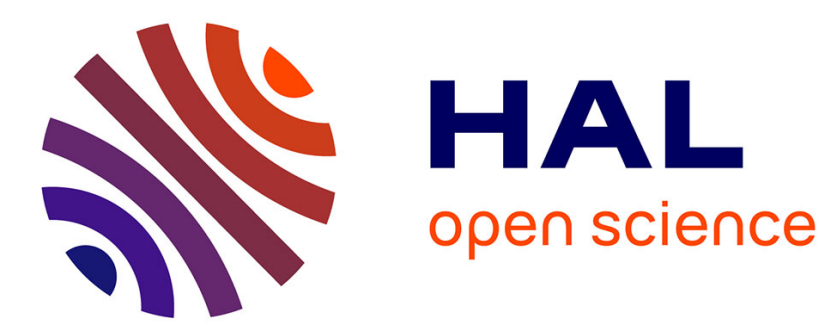

\title{
Spectromètre à réseau à exploration rapide
}

\author{
H. Chantrel, M. Denis, A. Baldy
}

\section{To cite this version:}

H. Chantrel, M. Denis, A. Baldy. Spectromètre à réseau à exploration rapide. Revue de Physique Appliquée, 1967, 2 (4), pp.245-248. 10.1051/rphysap:0196700204024500 . jpa-00242799

\section{HAL Id: jpa-00242799 https://hal.science/jpa-00242799}

Submitted on 1 Jan 1967

HAL is a multi-disciplinary open access archive for the deposit and dissemination of scientific research documents, whether they are published or not. The documents may come from teaching and research institutions in France or abroad, or from public or private research centers.
L'archive ouverte pluridisciplinaire HAL, est destinée au dépôt et à la diffusion de documents scientifiques de niveau recherche, publiés ou non, émanant des établissements d'enseignement et de recherche français ou étrangers, des laboratoires publics ou privés. 


\title{
SPEGTROMÈTRE A RÉSEAU A EXPLORATION RAPIDE
}

\author{
Par H. GHANTREL, M. DENIS et A. BALDY, \\ Faculté des Sciences Saint-Jérôme, Marseille (1 $\left.3^{\mathrm{e}}\right)$.
}

Résumé. - Un spectromètre à réseau plan est étudié et réalisé, qui permet l'exploration rapide (jusqu'à $3 \AA / \mu$ s) d'une large bande spectrale (jusqu'à $5000 \AA$ d'étendue) avec une résolution effective de $5 \AA$ environ. On donne la description du dispositif permettant une telle exploration, ainsi que quelques spectres illustrant les possibilités de l'appareil.

Abstract. - A grating spectrometer has been designed and built which allows rapid scanning rates (over $3 \AA / \mu \mathrm{s}$ ) over a wide spectral range (about $5000 \AA$ wide with a 600 lines per millimeter grating) with an effective resolving power of $5 \AA$. The basic principles of the instrument are described together with some spectra illustrating its capabilities.

I. Introduction. - L'appareil est destiné à suivre l'évolution du spectre de phénomènes durant de quelques secondes à quelques millisecondes. Certaines réalisations permettent déjà de telles études [1], [2], [3]. La nouvelle solution est conçue de manière à concilier au mieux, sans complications excessives, les impératifs habituels, à savoir : recherche de la luminosité maximale compatible avec la résolvance imposée, d'où l'utilisation d'un réseau; possibilité d'exploration d'un vaste domaine spectral, d'où l'optique à miroirs; faible temps mort entre deux explorations successives; vitesse d'exploration réglable dans une large bande de vitesses; faible altération de la fonction statique d'appareil à toutes les vitesses possibles d'exploration, par une construction qui élimine les vibrations mécaniques.

L'appareil présente une disposition du réseau et des fentes analogue à celle du spectrographe de Jarrel [4]. Il comporte en outre un dispositif d'exploration rapide constitué par deux groupes de miroirs plans, mobiles, parallèles aux traits du réseau : un groupe de miroirs explorateurs et un groupe de miroirs suiveurs. Chacun de ces derniers intervient à tour de rôle pour réfléchir suivant une direction fixe le faisceau sélectionné par l'un des premiers. L'un des miroirs suiveurs est donc associé à l'un des miroirs explorateurs pendant la durée d'une exploration. L'exploration suivante fait intervenir une nouvelle paire de miroirs plans, et ainsi de suite. Le tout est disposé et dimensionné de manière à fonctionner sans jamais limiter l'étendue de faisceau correspondant au plein éclairement du réseau.

II. Description de l'appareil. - A. Description DU MONTAGE OPTIQUE. - Les miroirs explorateurs doivent circuler dans le plan de dispersion du réseau. Pour cela, ils sont fixés à intervalles réguliers sur le contour d'un support circulaire mobile par rotation autour d'un axe $X^{\prime} X$ parallèle aux traits du réseau. Cet axe est vertical pour assurer un meilleur équilibrage dynamique des couronnes et une meilleure répartition des efforts sur les pivots. Les miroirs suiveurs sont eux aussi disposés en couronne sur un autre support circulaire coaxial au premier, mais situé au-dessus, de plus petit rayon et tournant dans le même sens à la vitesse angulaire moitié.

La figure 1 représente une coupe verticale schématique de l'appareil suivant l'axe $\mathrm{X}^{\prime} \mathrm{X}$. On peut $\mathrm{y}$

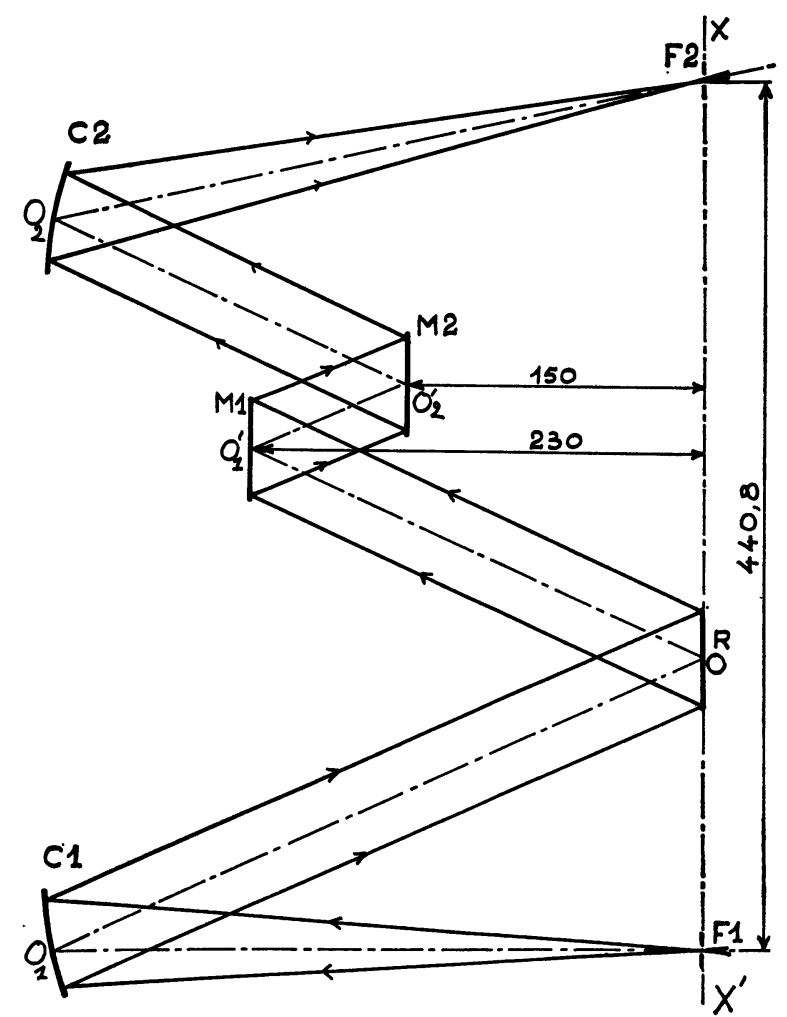

FIG. 1. - Coupe verticale schématique. 
suivre en projection la marche des faisceaux. La fente source $F_{1}$ est verticale. Nous l'avons placée suivant l'axe de rotation de l'appareil par commodité, mais elle peut être placée ailleurs. $F_{1}$ est au foyer du miroir concave fixe $\mathrm{C}_{\mathbf{1}}(\varnothing 50 \mathrm{~mm} ; f=316 \mathrm{~mm}) . \mathrm{C}_{1}$ renvoie donc un faisceau parallèle sur le réseau $R$ qui est totalement éclairé. L'axe vertical du réseau coïncide avec l'axe de rotation $\mathrm{X}^{\prime} \mathrm{X}$, bien que ce ne soit pas non plus essentiel. Au rayon moyen incident sur $R$ correspond maintenant une famille de rayons moyens réfléchis, dispersés en longueur d'onde suivant une portion de plan inclinée sur l'axe $\mathrm{X}^{\prime} \mathrm{X}$ et qu'elle coupe au centre $O$ du réseau. L'un d'eux, qui correspond donc à une longueur d'onde $\lambda_{0}$ bien définie, rencontre le centre $\mathrm{O}_{1}^{\prime}$ du miroir plan $\mathrm{M}_{1}$. L'ensemble des rayons parallèles à $\mathrm{OO}_{1}^{\prime}$ constitue le faisceau qui convergera au centre de la fente de sortie après réflexion sur le miroir plan suiveur $\mathrm{M}_{2}$ et sur le miroir concave $\mathrm{C}_{2}$. Tous les rayons associés au rayonnement de longueur d'onde $\lambda_{0}$ contribueront ainsi à former en $F_{2}$ l'image de $F_{1}$. A toute autre longueur d'onde correspond une autre image de $F_{1}$ située en dehors de la fente $F_{2}$.

La figure 2 est une vue de dessus schématique de l'appareil.

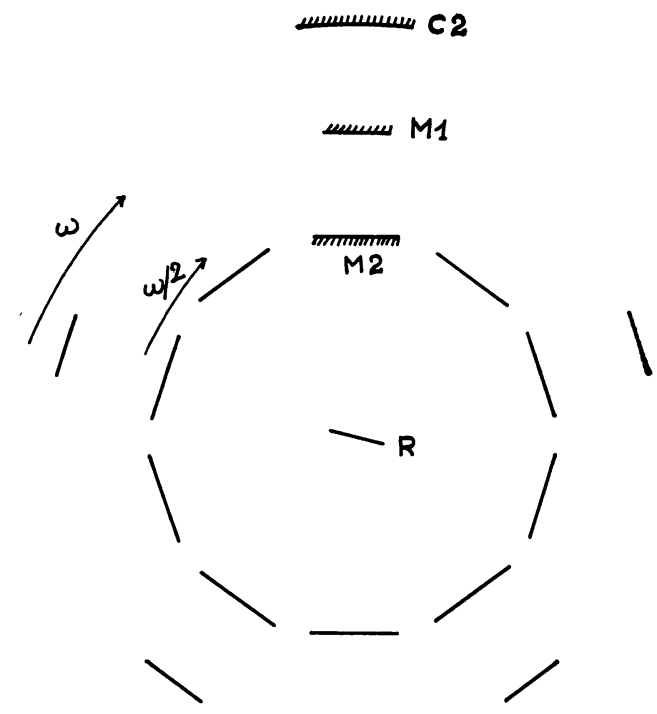

FIG. 2. - Vue de dessus schématique.

La figure 3 montre comment, du fait que la vitesse angulaire de la couronne portant les miroirs explorateurs est double de celle qui porte les miroirs suiveurs, ces derniers transmettent les rayons destinés à la fente de sortie suivant une direction fixe. On remarquera que les miroirs $\mathbf{M}_{2}$ doivent être plus larges que les miroirs $\mathbf{M}_{1}$ pour ne pas limiter l'étendue du faisceau en cours d'exploration (ils ont $50 \mathrm{~mm}$ de haut comme les miroirs $M_{1}$ mais $70 \mathrm{~mm}$ de large). Pour la même raison, le miroir concave $\mathrm{C}_{2}$ doit être
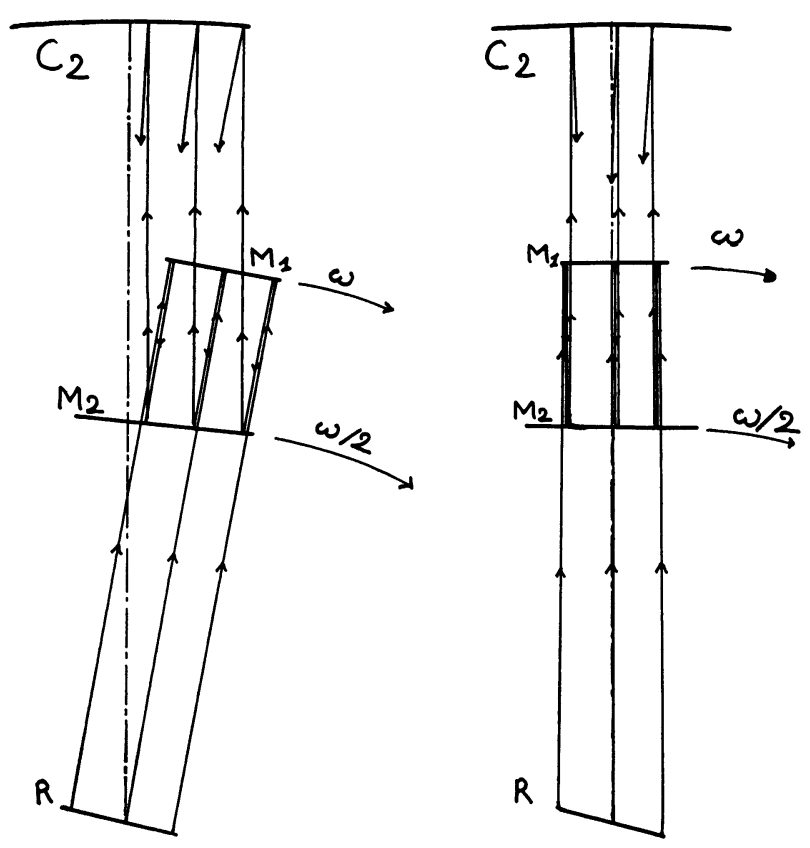

FIG. 3. - Détail de l'exploration.

plus large que $\mathrm{C}_{1}(\varnothing=100 \mathrm{~mm})$. On remarquera aussi que, lorsque $M_{1}$ a fait un tour complet, le miroir suiveur $M_{2}$ qui lui était associé dans la précédente exploration n'a fait qu'un demi-tour. Il faut donc associer à chaque miroir $M_{1}$ deux miroirs $M_{2}$ diamétralement opposés pour que $M_{1}$ travaille à chaque passage. Ainsi s'explique qu'il y ait deux fois plus de miroirs suiveurs que de miroirs explorateurs. Comme les miroirs suiveurs $M_{2}$ sont plus grands et deux fois plus nombreux que les miroirs $M_{1}$, et comme ils sont répartis sur un support de circonférence plus petite que celle du support des miroirs $M_{1}$, c'est leur encombrement qui limite leur nombre. Notre choix de cinq miroirs $M_{1}$ et dix miroirs $M_{2}$ est le résultat d'un compromis entre le souci de réduire le plus possible les temps morts entre explorations successives, de ne pas trop réduire la bande spectrale explorée et de ne pas exagérer les dimensions de l'appareil de manière à faire tourner vite les couronnes sans danger.

La conception générale de l'appareil, et notamment la nécessité d'introduire des miroirs suiveurs, est justifiée par ailleurs [5]. Cette étude qui comporte aussi une analyse chiffrée des mérites respectifs des solutions connues ne sera pas reprise ici.

B. Observation des spectres. - Le flux lumineux est reçu sur un photomultiplicateur EMI relié à un oscilloscope Tektronix par une résistance de charge de $22 \mathrm{k} \Omega$.

Pour corriger les défauts de construction résiduels 
qui subsistent nécessairement, défauts qui se traduisent par de petits écarts latéraux du signal observé sur l'écran d'un oscilloscope lorsqu'on se contente de synchroniser la fréquence de balayage de l'écran de l'oscilloscope avec la rotation des miroirs, nous déclenchons ce balayage par l'exploration spectrale elle-même. Le spectre lui-même ne peut être utilisé comme signal de déclenchement puisqu'il évolue. Nous avons donc utilisé une source auxiliaire dont le faisceau évite le réseau, et qui, par réflexion sur $M_{1}$, $\mathrm{M}_{2}$ et $\mathrm{C}_{2}$, donne une image proche de $\mathrm{F}_{2}$ juste avant le défilement du spectre devant $\mathrm{F}_{2}$. Gette image éclaire une cellule $\mathrm{PHG}_{1}$ dont le signal amplifié traverse un circuit de mise en forme destiné à raidir le front de déclenchement du balayage de l'écran de l'oscilloscope. Le diagramme de l'ensemble de l'appareillage est représenté sur la figure 4 .

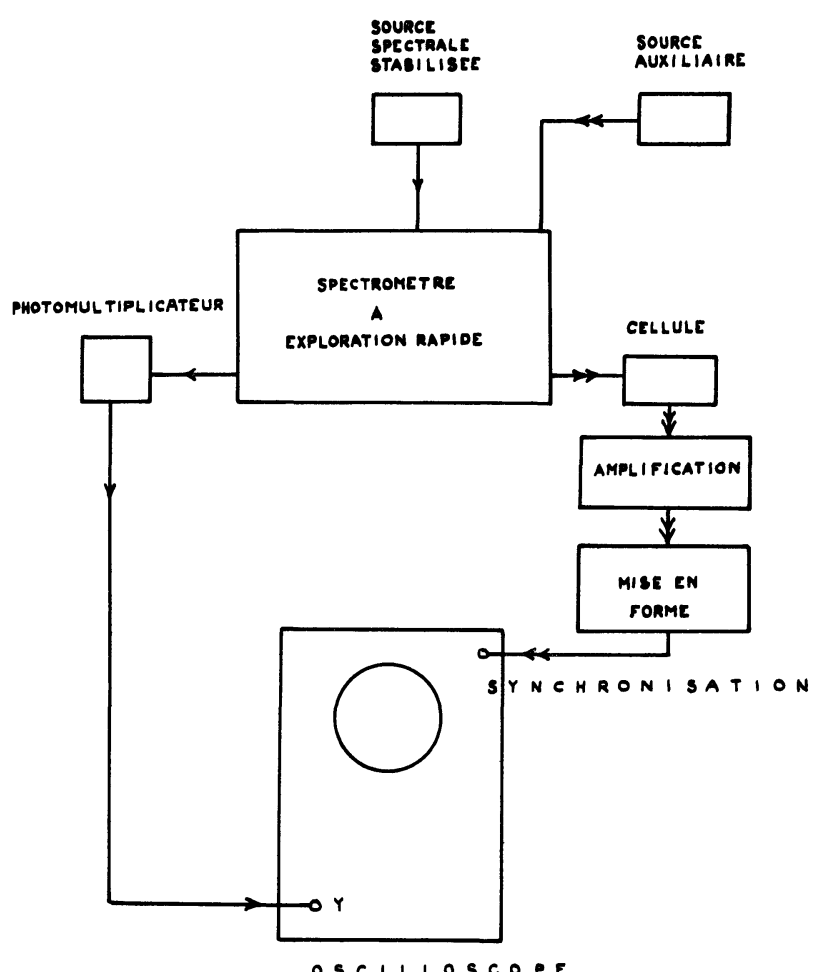

FIG. 4.

Diagramme de l'appareillage complet.

III. Résultats. - La figure 5 montre le spectre continu d'une lampe à filament de tungstène fonctionnant sous $6 \mathrm{~V}$ et $5 \mathrm{~A}$. La haute tension appliquée au photomultiplicateur est de $1000 \mathrm{~V}$. Le réseau utilisé a 600 traits par millimètre. Le temps de pose correspond à une soixantaine d'explorations successives, ce qui permet d'apprécier la stabilité du résultat en position comme en intensité, bien que dix explorations spectrales consécutives utilisent dix chemins optiques différents. La vitesse d'exploration est de

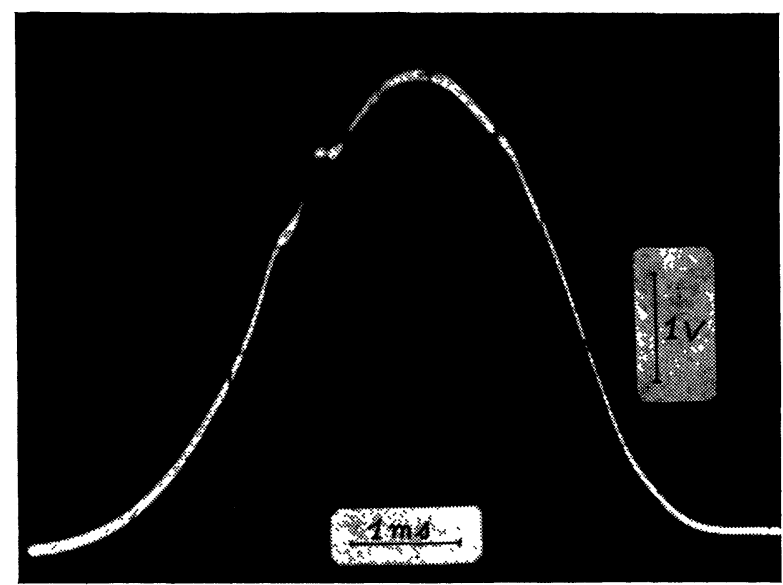

FIG. 5.

Spectre d'une lampe à filament de tungstène·

$0,3 \AA / \mu$ s, soit une vitesse de 3 tours par seconde pour les miroirs $M_{1}$.

La figure 6, représente le spectre d'une lampe spectrale Philips $\mathrm{ZnCdHg}$, alimentée en courant alternatif. La haute tension appliquée au photomultiplica-

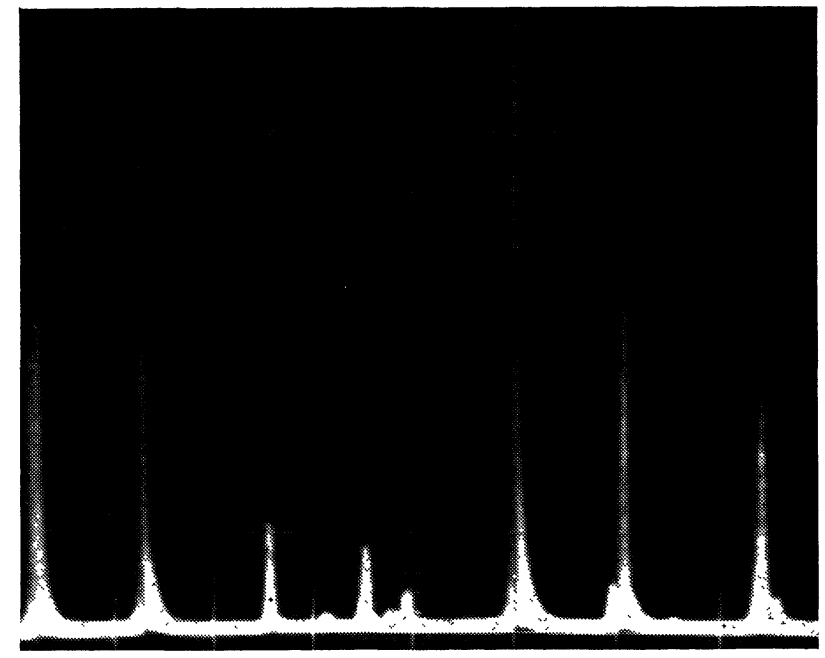

FIG. 6. - Spectre d'une lampe Philips $\mathrm{ZnCdHg}$.

Sur l'écran, l'amplitude est de $2 \mathrm{~V} / \mathrm{cm}$ et la vitesse de balayage de $1 \mathrm{~ms} / \mathrm{cm}$.

teur est de $900 \mathrm{~V}$ et le réseau utilisé a 600 traits par millimètre. Le temps de pose correspond aussi à une soixantaine d'explorations successives. La vitesse d'exploration est de $0,3 \AA / \mu$ s.

La figure 7 est constituée par le doublet jaune du mercure $(5770 \AA, 5790 \AA$ ) enregistré avec un réseau de 1200 traits par millimètre et des fentes de largeur de $0,04 \mathrm{~mm}$, à la vitesse de $0,3 \AA / \mu \mathrm{s}$. 


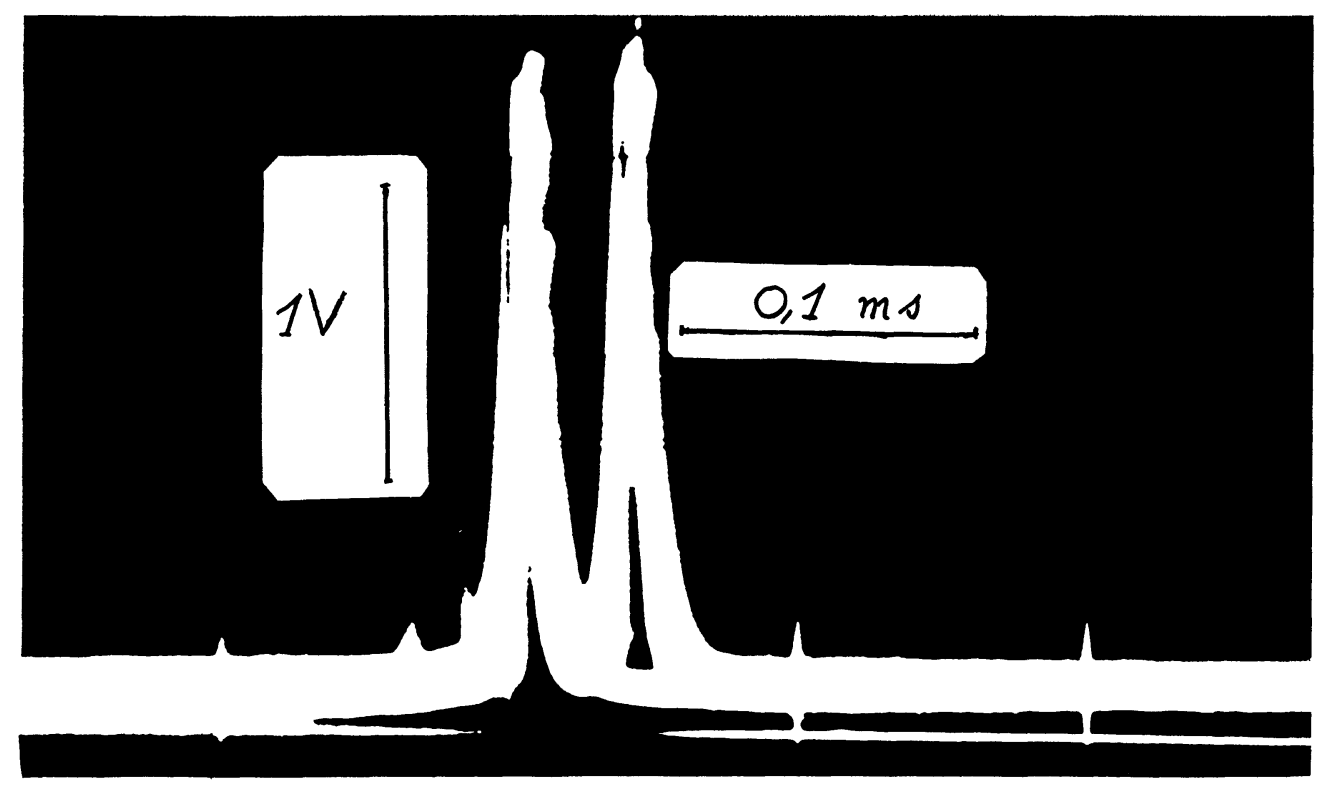

FIG. 7. - Doublet jaune du mercure.

Sur l'écran, l'amplitude est de $1 \mathrm{~V} / \mathrm{cm}$ et la vitesse de balayage de $0,1 \mathrm{~ms} / \mathrm{cm}$.

IV. Conclusion. - Le prototype réalisé est un spectromètre à exploration rapide $\left(v_{\max } \simeq 3 \AA / \mu \mathrm{s}\right)$ mais non ultrarapide comme ceux de Church et Gampel [1] et Hill et Beckner [2] $\left(v_{\max }=200 \AA / \mu \mathrm{s}\right)$. On peut, sur le même principe, réaliser un appareil bien plus rapide en diminuant ses dimensions. On peut réduire la largeur des miroirs suiveurs, ce qui revient à réduire la bande spectrale explorée. On peut surtout réduire le nombre des miroirs explorateurs, ce qui revient à augmenter la proportion des temps morts.

Les fluctuations d'intensité imputables à l'exploration sont d'environ $2 \%$. Les vibrations mécaniques résiduelles produisent une fluctuation de l'ordre de $\pm 5 \AA$ dans la position d'une raie sur l'écran de l'oscilloscope à la vitesse maximale d'exploration. soit $v_{\max }=3 \AA / \mu \mathrm{s}$, ce qui correspond à une vitesse de rotation de 30 tours par seconde pour les miroirs $M_{1}$. Il est possible de réduire notablement ces vibrations.

La grande luminosité de ce spectromètre le rend particulièrement apte à l'étude de phénomènes de faible intensité. Il est destiné à des études biochimiques.

La partie mécanique de cet appareil a été étudiée et réalisée par la Société Terrin Technique Industrie de Marseille à laquelle nous adressons nos vifs remerciements.

Get appareil a fait l'objet d'un dépôt de Brevet enregistré sous le no 21911 à la Préfecture de Marseille.

Manuscrit reçu le 25 juillet 1967.

\section{BIBLIOGRAPHIE}

[1] Church (Ch.) et Gampei (L.), Applied Optics, février 1966, 241-4.

[2] HILI, et BECKNER, Applied Optics, 1964, 229-232.

[3] Niesel, (W.), Lubbers (D. W.), SchNeEWolf (D.),
RICHTER et BOTTICHER, Rev. Sci. Inst., 1964, 35, 578-581.

[4] Richard (F.), JaRrei, J. Optical Soc. Amer., 1955, 45-4, 259 à 269.

[5] DENIS (M.), Thèse de spécialité, Marseille, 1967. 